\title{
A High-Speed Four-Transmitter Four-Receiver MIMO OFDM Testbed: Experimental Results and Analyses
}

\author{
Weidong Xiang, ${ }^{1}$ Paul Richardson, ${ }^{1}$ Brett Walkenhorst, ${ }^{2}$ Xudong Wang, ${ }^{3}$ and Thomas Pratt $^{2}$ \\ ${ }^{1}$ ECE Department, University of Michigan-Dearborn, 126 ELB, 4901 Evergreen Rd., Dearborn, MI, 48128, USA \\ ${ }^{2}$ Communications and Networking Division, Information Technology and Telecommunications Laboratory, \\ Georgia Tech Research Institute, Atlanta, GA 30332-0832, USA \\ ${ }^{3}$ Kiyon Company, 4225 Executive Square, Suite 290, La Jolla, CA 92037, USA
}

Received 30 November 2004; Revised 1 September 2005; Accepted 1 September 2005

By adopting multiple-input multiple-output (MIMO) and orthogonal frequency-division multiplexing (OFDM) technologies, indoor wireless systems could reach data rates up to several hundreds of Mbits/s and achieve spectral efficiencies of several tens of bits/Hz/s, which are unattainable for conventional single-input single-output systems. The enhancements of data rate and spectral efficiency come from the fact that MIMO and OFDM schemes are indeed parallel transmission technologies in the space and frequency domains, respectively. To validate the functionality and feasibility of MIMO and OFDM technologies, we set up a fourtransmitter four-receiver OFDM testbed in a typical indoor environment, which achieves a peak data rate of $525 \mathrm{Mbits} / \mathrm{s}$ and a spectral efficiency of $19.2 \mathrm{bits} / \mathrm{Hz} / \mathrm{s}$. The performances including MIMO channel characteristics, bit-error rate against signal-tonoise ratio curves, the impairments of carrier frequency offset and channel estimation inaccuracy, and an asymmetric MIMO scheme are reported and analyzed in this paper.

Copyright ( 2006 Hindawi Publishing Corporation. All rights reserved.

\section{INTRODUCTION}

Combination of multiple-input multiple-output (MIMO) and orthogonal frequency-division multiplexing (OFDM) technologies enables wireless communications systems to easily exceed the maximum intersymbol interference (ISI) free data rate, which equals the reciprocal of maximum excess delay of the wireless channel the signal passing through. Bell Laboratory layered space-time (BLAST) scheme is a common used MIMO technology, which sends independent user information over multiple antennas at the same frequency and bandwidth simultaneously. MIMO systems adopting BLAST scheme can reach spectral efficiencies of several tens of bits/Hz/s [1], which are unattainable for conventional single-input single-output (SISO) systems. The secret is that MIMO systems deliver information in parallel in the spacedomain. On the other hand, OFDM is a parallel transmission technology in the frequency domain, which delivers information over a set of orthogonal subcarriers. The number of subcarriers is deliberately selected to allow each subcarrier to experience flat fading. Furthermore, OFDM systems efficiently eliminate the ISI by the use of cyclic prefix (CP). When adopting the two parallel transmission technologies, an indoor wireless link could offer data rates much greater than those that defined by current wireless local areas network (WLAN) standards.

The authors have reported a three-transmitter threereceiver $(3 \times 3)$ MIMO testbed offering a data rate of 281.25 Mbits/s [2] and a real-time two-transmitter tworeceiver $(2 \times 2)$ space-time coding MIMO testbed reaching a data rate of $30 \mathrm{Mbits} / \mathrm{s}$ [3]. Additionally, there are several MIMO testbeds in recent literatures [1, 4-8]. Bell Laboratory realized a $8 \times 12 \mathrm{MIMO}$ testbed achieving a spectrum efficiency of $25.9 \mathrm{bit} / \mathrm{Hz} / \mathrm{s}$ at $1.9 \mathrm{GHz}$ with $30 \mathrm{KHz}$ bandwidth [1]. The Iospan wireless company established a $2 \times 3$ MIMO broadband wireless prototype offering a data rate of 13.6 Mbits/s [4]. The University of Bristol completed a $4 \times 6$ MIMO testbed at $5 \mathrm{GHz}$ realizing a data rate of $96 \mathrm{Mbits} / \mathrm{s}$ [5]. The Motorola company finished a $2 \times 2 \mathrm{MIMO}$ testbed at $3.65 \mathrm{GHz}$ offering a data rate of $180 \mathrm{Mbits} / \mathrm{s}$ [6]. The Brigham Young University (BYU) developed a real-time $4 \times 4$ MIMO testbed using multiple TMS320C6203 DSPs and achieving a data rate of $4 \mathrm{Mbits} / \mathrm{s}$ [7]. Table 1 compares the key parameters and specifications of the above MIMO testbeds.

The main contributions of this paper are the presentation of the measured bit-error rate (BER) versus signal-tonoise ratio (SNR) curves, the comparison of the experimental data with simulation results based on the indoor MIMO 
TABLE 1: The comparisons of recently reported MIMO testbeds.

\begin{tabular}{|c|c|c|c|c|c|c|}
\hline Name & $\begin{array}{c}\text { MIMO } \\
\text { configuration }\end{array}$ & Data rate & Frequency/bandwidth & $\begin{array}{l}\text { Spectral } \\
\text { efficiency }\end{array}$ & Modulation & $\begin{array}{c}\text { Completed } \\
\text { year }\end{array}$ \\
\hline $\begin{array}{l}\text { Georgia Tech } \\
\text { testbed \#1 [2] }\end{array}$ & $3 \times 3$ & $281.25 \mathrm{Mbits} / \mathrm{s}$ & $2.435 \mathrm{GHz} / 19.5 \mathrm{MHz}$ & $14.4 \mathrm{bits} / \mathrm{Hz} / \mathrm{s}$ & 64-QAM/OFDM & 2004 \\
\hline $\begin{array}{l}\text { Georgia Tech testbed \#2 } \\
\text { [3] (real-time mode) }\end{array}$ & $\begin{array}{l}2 \times 2 \text { (space- } \\
\text { time coding })\end{array}$ & $30 \mathrm{Mbits} / \mathrm{s}$ & $2.435 \mathrm{GHz} / 6.25 \mathrm{MHz}$ & $4.8 \mathrm{bits} / \mathrm{Hz} / \mathrm{s}$ & 64-QAM/OFDM & 2002 \\
\hline $\begin{array}{l}\text { Bell Laboratory } \\
\text { testbed [1] }\end{array}$ & $8 \times 12$ & 777.6 Kbits/s & $1.9 \mathrm{GHz} / 30 \mathrm{KHz}$ & $25.92 \mathrm{bits} / \mathrm{Hz} / \mathrm{s}$ & 16-QAM & 1999 \\
\hline $\begin{array}{l}\text { Iospan wireless } \\
\text { testbed [4] }\end{array}$ & $2 \times 3$ & 13.6 Mbits/s & $2.5-2.6 \mathrm{GHz} / 2 \mathrm{MHz}$ & $6.8 \mathrm{bits} / \mathrm{Hz} / \mathrm{s}$ & 64-QAM/OFDM & 2002 \\
\hline $\begin{array}{l}\text { University of Bristol } \\
\text { testbed [5] }\end{array}$ & $4 \times 6$ & $96 \mathrm{Mbits} / \mathrm{s}$ & $5 \mathrm{GHz} / 12 \mathrm{MHz}$ & $8 \mathrm{bits} / \mathrm{Hz} / \mathrm{s}$ & QPSK/OFDM & 2001 \\
\hline $\begin{array}{l}\text { Motorola } \\
\text { testbed [6] }\end{array}$ & $2 \times 2$ & $180 \mathrm{Mbits} / \mathrm{s}$ & $3.65 \mathrm{GHz} / 20 \mathrm{MHz}$ & $9 \mathrm{bits} / \mathrm{Hz} / \mathrm{s}$ & 64-QAM/OFDM & 2001 \\
\hline $\begin{array}{l}\text { BYU testbed [7] } \\
\text { (real-time mode) }\end{array}$ & $4 \times 4$ & $2 \mathrm{Mbits} / \mathrm{s}$ & $2.45 \mathrm{GHz} / 250 \mathrm{KHz}$ & $8 \mathrm{bits} / \mathrm{Hz} / \mathrm{s}$ & QPSK & 2001 \\
\hline
\end{tabular}

channel model given by IEEE 802.11 study group [9], and the exploration of the impairments of carrier frequency offset and channel estimation inaccuracy. We also propose an asymmetric MIMO scheme to efficiently enhance the robustness of MIMO wireless links. This work is a continuation of [2]. In [2], we focused on the configuration of the testbed, time, and frequency synchronizations, and BLAST demodulation algorithms.

In addition, we increase the sample rate of baseband single from 25 mega-samples per second (MSPS) to 35 MSPS and upgrade the MIMO configuration from $3 \times 3$ to fourtransmitter four-receiver $(4 \times 4)$. Then the upgraded testbed achieves a data rate of $525 \mathrm{Mb} / \mathrm{s}$ and a spectral efficiency of $19.2 \mathrm{bits} / \mathrm{Hz} / \mathrm{s}$.

This paper is arranged as follows. Section 2 briefly reviews the system design, the configuration of the testbed, and the experiments. Section 3 studies the characteristics of $4 \times 4$ MIMO channel by exploring the condition number of MIMO channel. The time variations of the MIMO channel are investigated as well. In Section 4, the measured BERSNR curves are presented and compared with the simulation results based on the MIMO channel model. We then discuss the degradation of the BER-SNR curves caused by channel estimation inaccuracy. In Section 5, we investigate the degradation of the BER-SNR curves caused by carrier frequency offset. To enhance the transmission robustness, Section 6 suggests adopting an asymmetric MIMO scheme, which decreases the performance sensitivity to the channel status. Conclusions are given finally.

\section{SYSTEM OVERVIEW AND THE EXPERIMENTS}

In order to demonstrate a high-speed indoor wireless link adopting MIMO and OFDM technologies, we set up a $4 \times 4$ testbed in the software radio laboratory at Georgia Institute of Technology in April 2004. The testbed runs in an offline mode which transmits and captures the signal in a real-time mode but processes it offline. Offline testbeds can efficiently validate the functions and performances of a wireless communication system with much simple implementations compared to real-time testbeds and thus have been widely used for research-oriented efforts.

The key specifications of the testbed are as follows. At first, we select a center frequency of $2.435 \mathrm{GHz}$ because of the available federal communications commission (FCC) license. Then we adopt the fast Fourier transformation (FFT) with a block size of 256. The baseband signals are sent in a rate of 35 MSPS. If the IEEE 802.11a based OFDM symbol configuration is used, the $\mathrm{CP}$ has a duration of 0.46 us (16 complex samples), which is less than the typical maximum excess delay of indoor channels, $0.8-1.2$ us. In order to extend the $\mathrm{CP}$ and keep a reasonable time domain overhead (the ratio of the length of CP to that of an OFDM symbol), we need to increase the FFT block size. However, the FFT complexity increases with its block size as well. Considering the above two factors, the FFT with a block size of 256 is selected to enlarge the CP duration to 1.8 us (64 complex samples), greater than the typical maximum excess delay of indoor channels. Meanwhile, the corresponding computation load is still acceptable. We further adopt the short and long preambles defined by the IEEE 802.16 standard due to the same block size, which are used to time and frequency synchronizations and channel estimation, respectively.

The data rate of a wireless communication system could be determined by multiplying the spectral efficiency of the modulation used and the bandwidth occupied. In the testbed, we transmit and receive the baseband signal at a sample rate of 35 MSPS, which implies the signal occupies a bandwidth of $35 \mathrm{MHz}$. To fit the FCC spectrum mask, 56 of 256 subcarriers are not used, which lead to a frequency domain overhead of $78.125 \%$ and reduce the signal bandwidth from $35 \mathrm{MHz}$ to $27.3438 \mathrm{MHz}$ [8]. As we know, pilots are normally used to track the variations of the channel state and carrier frequency offset after they have been initially estimated by using the preambles. These are designed for the highly variable channel environments. Since the fast 


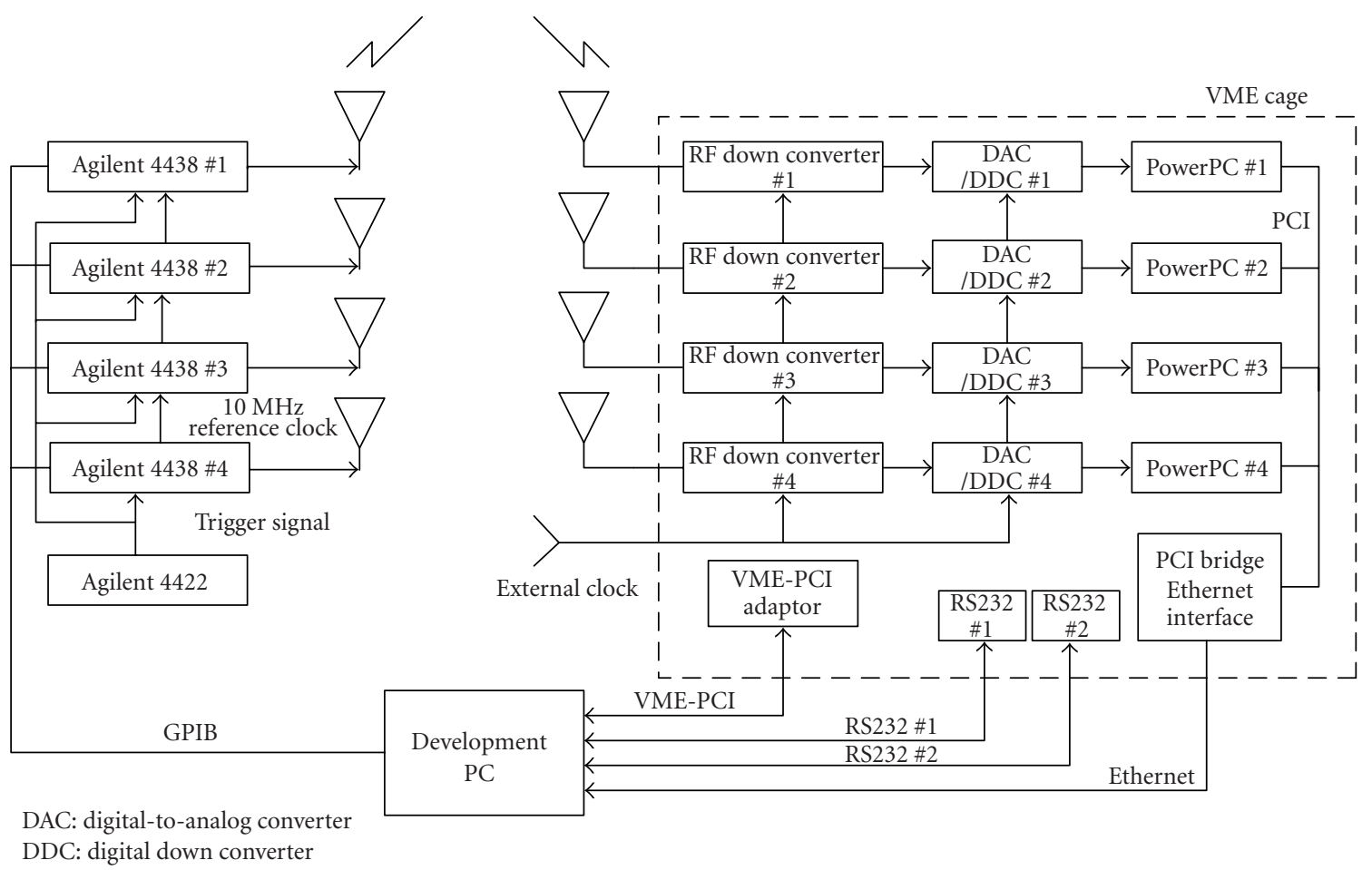

FIgure 1: The configuration of a $4 \times 4$ MIMO OFDM testbed.

baseband signal sample rate and the short fixed OFDM frame (45 OFDM symbols) adopted in the testbed, the channel state and frequency offset during one OFDM frame period are regarded as invariable. We then adopt all 8 pilots for data transmission and increase the data rate by about $4 \%$. (Even pilots are also intended for tracking the phase noise. We ignore its impairments since our testbed use Agilent signal generators which have very low phase noise.)

In the meantime, the overhead in time domain due to the use of CP is $256 / 320=80 \%$, where an OFDM symbol has 320 samples including a 64-sample CP. The testbed also adopts a $4 \times 4$ configuration offering four times data rate compared to a SISO system and the 64 quadrature amplitude modulation (QAM). Finally, the actual peak data rate is $35 \times 6 \times 4 \times 200 / 256 \times 256 / 320=525 \mathrm{Mbits} / \mathrm{s}$ and the spectrum efficiency is $19.2 \mathrm{bits} / \mathrm{Hz} / \mathrm{s}$.

The configuration of the testbed, shown in Figure 1, consists of four synchronized transmitters and four synchronized receivers. At the transmitters, four Agilent ESG4438C signal generators are employed to synchronously generate four independent OFDM frames, each of which consists of one short preamble, four long preambles used to MIMO channel estimation and 40 payload symbols. The OFDM frames are preloaded to the memories of the signal generators and are sent either in a burst mode or a continuous mode. A trigger signal provided by an Agilent ESG4422 signal generator is used to initiate the MIMO transmission. The receivers include low noise amplifies, RF down converters, analog-to-digital converters (ADC), digital down converters and PowerPC processors. An external clock is employed to allow the four receivers to work synchronously. Four RF signals from four receive antennas are down converted and then sampled by four ADCs. The sampled baseband signals are passed to a computer as four individual data files via an Ethernet interface. A MIMO OFDM demodulation program is applied to recover the four independent user data streams. The demodulation processing includes time synchronization, frequency synchronization, channel estimation, FFT, BLAST demodulation, and 64-QAM demapping. Their performances and computation loads are discussed in [2].

The experiments were conducted in the second floor of the Georgia Centers for Advanced Telecommunications Technologies building located at 250 14th street, Atlanta, Georgia. Figures 2 and 3 show the pictures of the transmitters and receivers. Two uniform linear antenna (ULA) arrays, consisting of four elements separated by three wavelengths, are mounted at the transmitters and receivers, respectively. Each element is a $2.4 \mathrm{GHz}$ dual polarized (horizontal polarization and vertical polarization, linear) omni-directional antenna covered by a radome. We select three typical locations to represent the common indoor wireless link scenarios. The first place represents a line-of-sight (LOS) wireless link within a typical laboratory. The second case is a nonLOS wireless link blocked by a wall and the third is a nonLOS wireless link from the laboratory to the corridor. All the three positions are shown in Figure 4. 


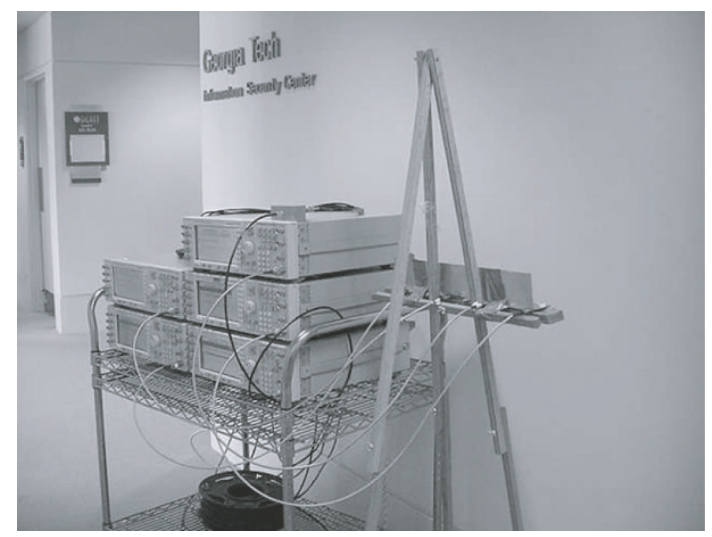

FIgURE 2: The transmitters of the $4 \times 4$ MIMO OFDM testbed.

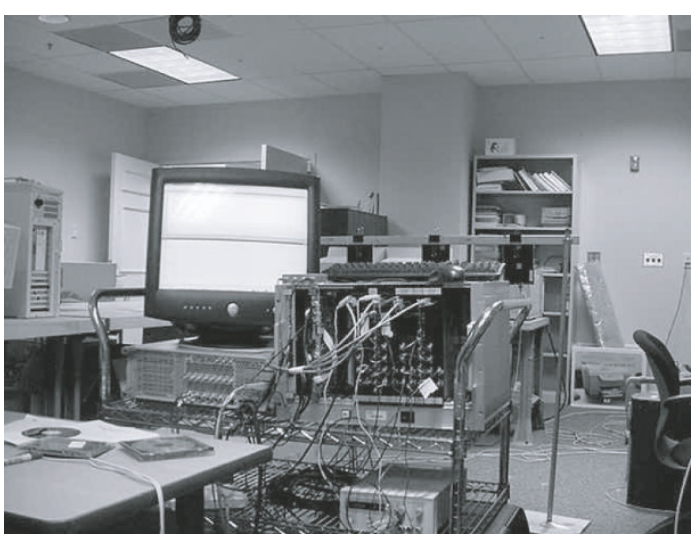

FIgure 3: The receivers of the $4 \times 4$ MIMO OFDM testbed.

\section{THE CHARACTERISTICS OF MIMO WIRELESS CHANNELS}

We allocate the whole $27.3438 \mathrm{MHz}$ bandwidth to 200 subcarriers and each subcarrier occupies $136.7 \mathrm{KHz}$ bandwidth, less than the coherence bandwidth of a typical indoor wireless channel. Then we assume that each subcarrier goes through a flat fading channel and an one-tap frequency equalizer for each subcarrier is sufficient to compensate the channel distortions.

For a $4 \times 4$ MIMO OFDM system, an OFDM symbol can be expressed as follows,

$$
r_{k}=H_{k} a_{k}+w_{k} \quad(k=1, \ldots, 200),
$$

where $r_{k}=\left[r_{1, k}, \ldots, r_{4, k}\right]^{T}, a_{k}=\left[a_{1, k}, \ldots, a_{4, k}\right]^{T}$, and $w_{k}=$ $\left[w_{1, k}, \ldots, w_{4, k}\right]^{T}$ are $4 \times 1$ receive signal, transmit signal, and Gaussian noise vectors. The elements, $r_{i, k}, a_{i, k}$, and $w_{i, k}$, $i=1, \ldots, 4$, represent the receive signal, transmit signal, and Gaussian noise at $i$ th antenna over $k$ th subcarrier, respectively. $H_{k}$ is a $4 \times 4$ matrix, of which the element $H_{i, j, k}$ $i, j=1, \ldots, 4$, represents the channel complex gain from the $j$ th transmitter to $i$ th antenna over $k$ th subcarrier. Normally, we have $E\left\{a_{k} a_{k}^{H}\right\}=\sigma_{s}^{2} I$, and $E\left\{w_{k} w_{k}^{H}\right\}=\sigma_{0}^{2} I$, where $I$ is

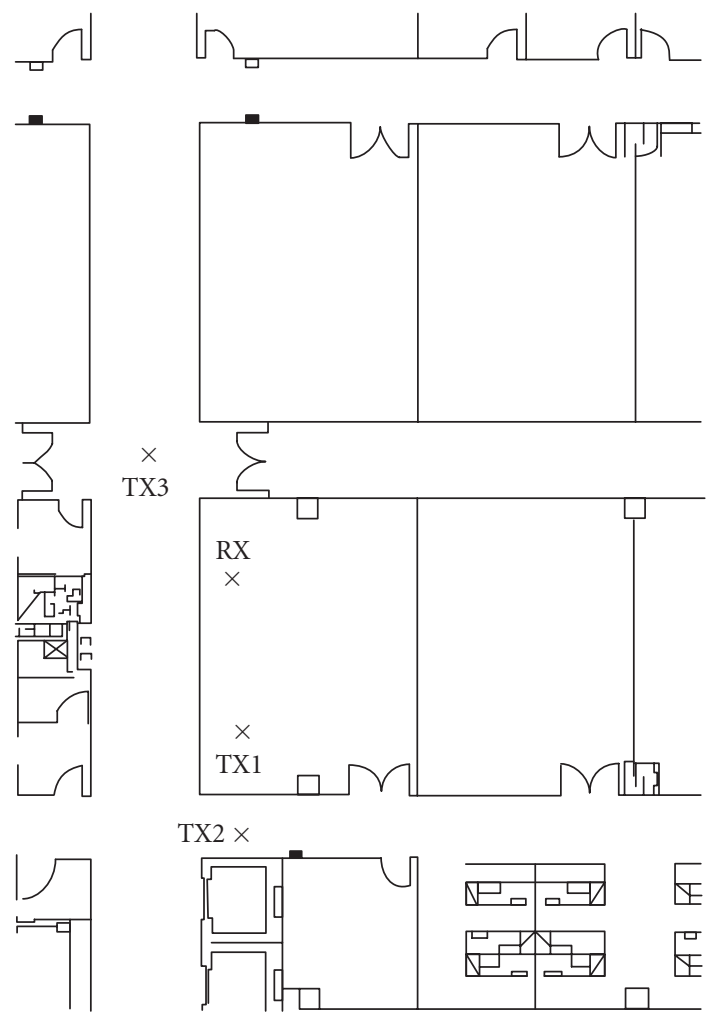

FIGURE 4: The floorplan of the building in which the LOS and NLOS measurements were made.

the $4 \times 4$ unit matrix and $(\cdot)^{H}$ represents conjugate transposition. $\sigma_{s}^{2}$ and $\sigma_{o}^{2}$ are the QAM symbols average power and noise variance. For 64-QAM modulation, we have $\sigma_{s}^{2}=42$.

The MIMO channel information is the decisive precondition for BLAST modulation. The MIMO channel is estimated by using four long preambles as the training signals. The training signals are configured to form a unitary matrix, expressed in the following equation. This configuration can significantly simplify the computations because time-consuming matrix inversion is replaced by simple matrix transposition.

$$
\left[\begin{array}{llll}
\operatorname{Tr}_{1,1} & \operatorname{Tr}_{1,2} & \operatorname{Tr}_{1,3} & \operatorname{Tr}_{1,4} \\
\operatorname{Tr}_{2,1} & \operatorname{Tr}_{2,2} & \operatorname{Tr}_{2,3} & \operatorname{Tr}_{2,4} \\
\operatorname{Tr}_{3,1} & \operatorname{Tr}_{3,2} & \operatorname{Tr}_{3,3} & \operatorname{Tr}_{3,4} \\
\operatorname{Tr}_{4,1} & \operatorname{Tr}_{4,2} & \operatorname{Tr}_{4,3} & \operatorname{Tr}_{4,4}
\end{array}\right]=\left[\begin{array}{rrrr}
1 & -1 & -1 & -1 \\
1 & 1 & 1 & -1 \\
1 & -1 & 1 & 1 \\
1 & 1 & -1 & 1
\end{array}\right] P l
$$

where $\operatorname{Tr}_{i, j}$ is the $j$ th training OFDM symbol at the $i$ th transmitter. $P l$ is the long preamble defined by the IEEE 802.16 standard [8].

Figure 5 shows an example of the measured 16-channel frequency response position 1. For comparison, the channel gains are normalized to eliminate the path loss. As we can see from the figure, the channel responses exhibit evident frequency selectivity.

In MIMO systems, the characteristics of the channel matrix decide the system capacity. Let $\rho_{i, k}, i=1,2,3,4$, represent the ordered singular values of the channel matrix at $k$ th 


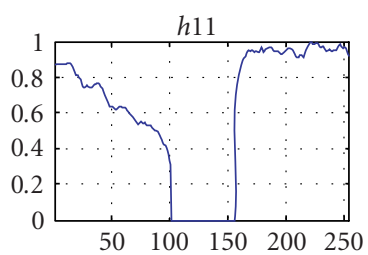

(a)

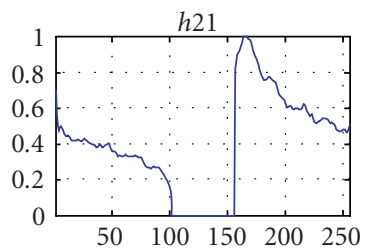

(e)

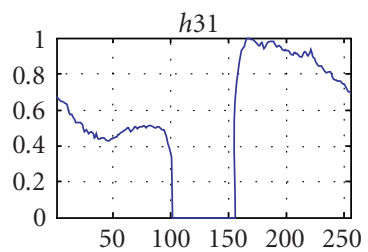

(i)

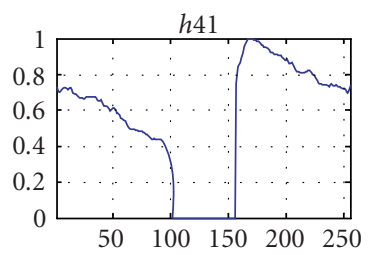

(m)

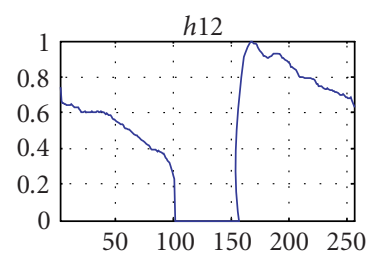

(b)

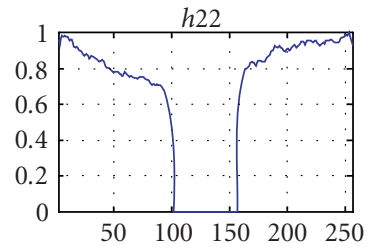

(f)

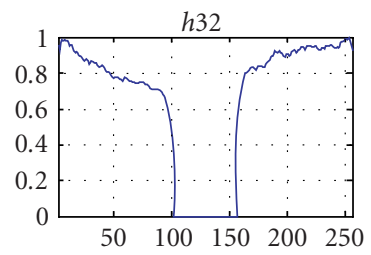

(j)

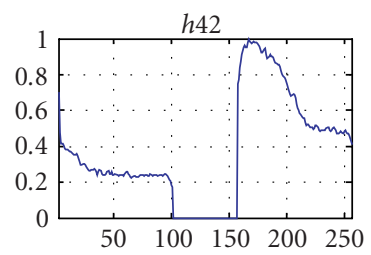

(n)

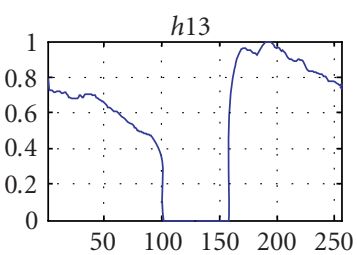

(c)

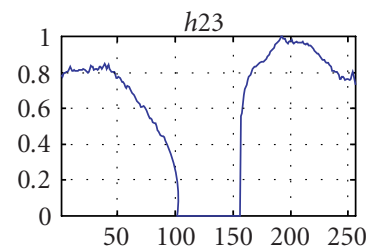

(g)

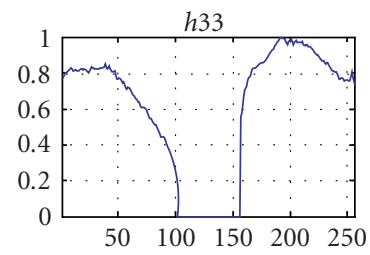

(k)

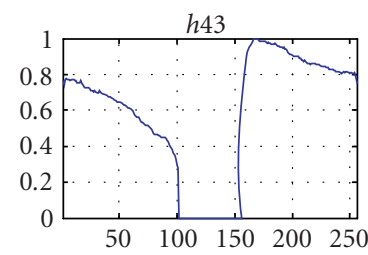

(o)

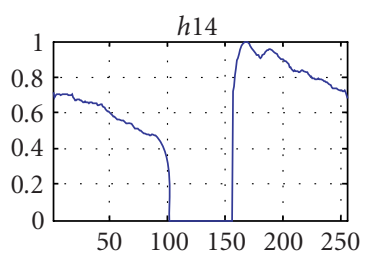

(d)

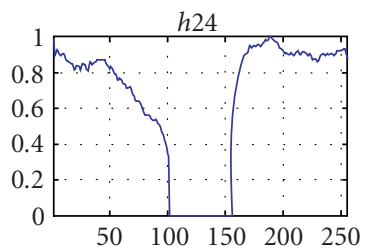

(h)

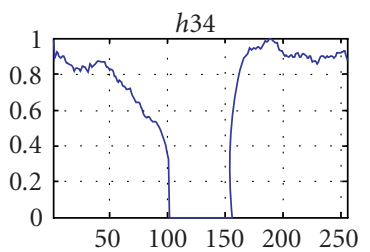

(1)

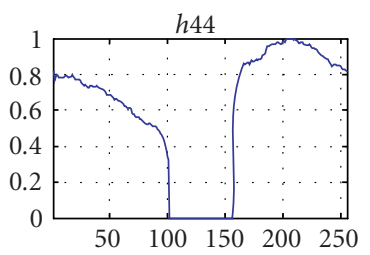

(p)

FIGURE 5: A measurement of 16-channel frequency responses at position 1.

subcarrier, that is, $\rho_{1, k} \geq \rho_{2, k} \geq \rho_{3, k} \geq \rho_{4, k}$. Then the condition number, $c_{k}$, at $k$ th subcarrier can be given as

$$
c_{k}=\frac{\rho_{1, k}}{\rho_{4, k}} .
$$

Figure 6 shows the condition numbers at different subcarriers associated with the MIMO channel shown in Figure 5. We define the average condition number over all the subcarriers as the condition number of a MIMO OFDM system, which is given by following equation,

$$
c=\frac{1}{200} \sum_{k=1}^{200} c_{k} .
$$

The capacity for $k$ th subcarrier and the total capacity over all subcarriers are given by

$$
\begin{gathered}
C_{k}=W_{k} \log _{2} \operatorname{det}\left(I_{4}+\frac{\mathrm{SNR}}{4} H_{k}{ }^{H} H_{k}\right), \\
C=\sum_{k=1}^{200} C_{k},
\end{gathered}
$$

where $W_{k}$ is the bandwidth of $k$ th subcarrier and SNR is the signal-to-noise ratio.

For a nonadaptive transmission, the channel status information is unknown to the transmitter and the transmitted power is evenly allocated to all the subcarriers. Under the condition, the MIMO channel with a lower condition number has a higher capacity. Particularly, when $H_{k}{ }^{H} H_{k}=4 I_{4}$, the capacity $\mathrm{C}$ reaches its maximum value. That is,

$$
C_{\max }=4 W \log _{2}(1+\mathrm{SNR})
$$

where the $W$ is the total bandwidth and $W=200 \times W_{k}$. For this case, the singular values are $\rho_{i, k}=2, i=1,2,3,4$, and the condition number is $c=1$. Figure 7 shows the simulation results and the fitting curve reflecting the variations of the normalized capacity, $C / C_{\max }$, against the channel condition numbers where the SNR $=30 \mathrm{~dB}$. The relationship between the channel condition number and normalized capacity is not unique and determined because several MIMO channel matrices could have similar condition numbers but different system capacities. Statistically, the MIMO channel 


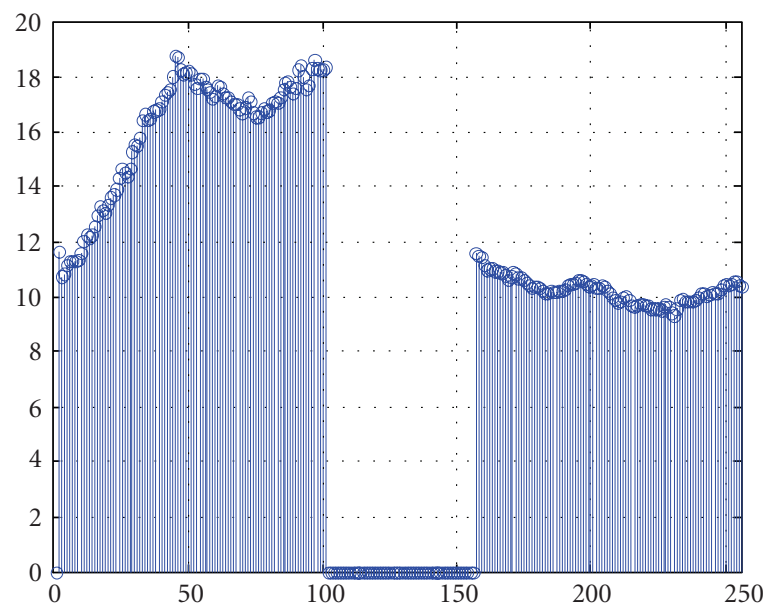

FIgURE 6: The condition numbers at different subcarriers for the MIMO channels at position 1.

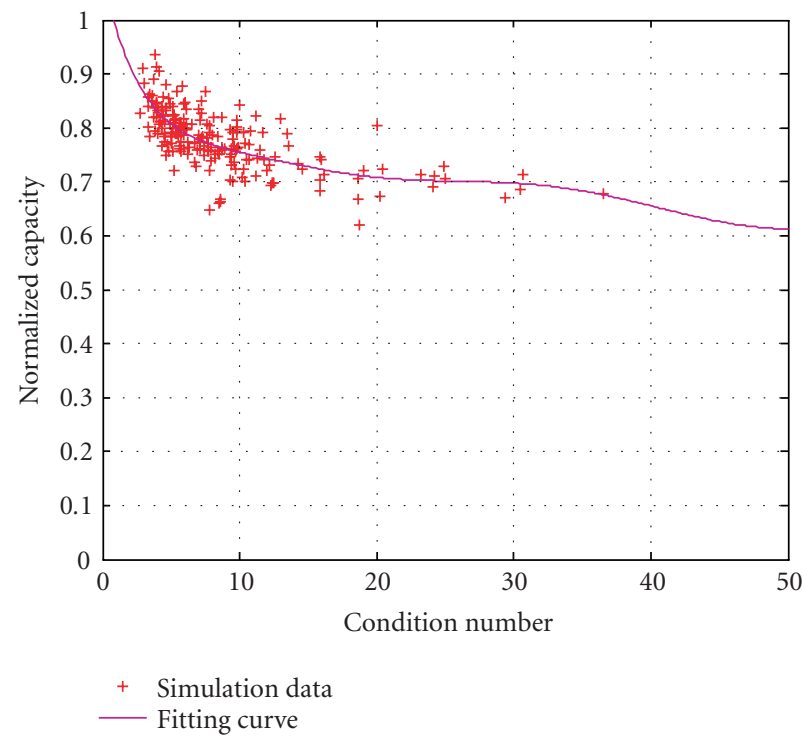

FIGURE 7: The distribution of the normalized capacities versus the channel condition numbers where the $\mathrm{SNR}=30 \mathrm{~dB}$.

with a condition number of 10 reaches about $75 \%$ of the maximum capacity.

Next, we observe the time variations of the MIMO channel. During the experiments, the transmitters and receivers are fixed while some pedestrians were moving around. Figure 8 shows the time variations of the condition numbers for the MIMO channels at the three different locations. In order to observe the MIMO channel variation in a period of several minutes, ten continuous MIMO channels are extracted and recorded in an interval of about one minute for each location. From Figure 8, we can see that the indoor channel variations are much lower compared to outdoor channels and mainly caused by the movements of the pedestrians and other inferences, like Bluetooth signals, and microwave oven leakage. The MIMO channels at position 1

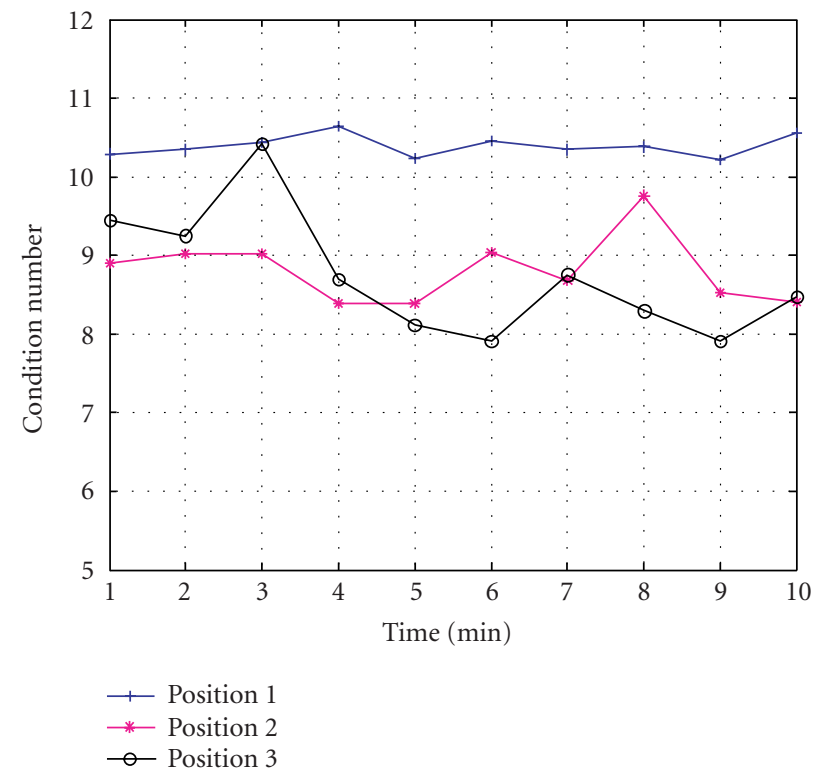

FIGURE 8: The variations of channel condition number measured at three different positions.

are with LOS link and have less variations comparing to position 2 and 3. On the other hand, the MIMO channels at position 2 and 3 have NLOS links and lower condition numbers meaning larger capacities.

\section{SYSTEM PERFORMANCE: BER-SNR CURVES}

The BER-SNR curve is a critical performance for a wireless communication system, which reflects the power efficiency. A SISO OFDM system with 64-QAM modulation has the same BER-SNR performance with single carrier system if the errors introduced by the FFT/inverse FFT (IFFT) processing are negligible. The BER-SNR curve of single carrier 64-QAM systems is given by the following equation:

$$
P_{b}=\frac{7}{12} Q\left(\sqrt{\frac{2}{7} \frac{E_{b}}{N_{0}}}\right)
$$

where $Q(x)=(1 / \sqrt{2 \pi}) \int_{x}^{\infty} e^{-t^{2} / 2} d t, P_{b}$ is the bit error probability, $E_{b}$ is signal energy per bit, and $N_{0}$ is power density of Gaussian noise. It is quite predicable that a $4 \times 4$ MIMO OFDM system has a much larger BER than a SISO OFDM system. The four transmitted signals are mixed with each other during the propagation. When one of them is separated during the demodulation, the others are presented as additional noise.

Figure 9 gives the measured BER-SNR curves of the $4 \times 4$ MIMO OFDM system at three locations shown in Figure 4. The SNR varies from $0 \mathrm{~dB}$ to $40 \mathrm{~dB}$, a reasonable upper bound for an actual wireless system. Ten trials are measured at each position. From the figure, we see that the $4 \times 4$ MIMO OFDM system presents a quite fair BER-SNR performance. This makes it imperative to adopt some advanced wireless transmission schemes, like powerful coding, diversity and adaptive modulation, to decrease the BER of MIMO wireless link. 


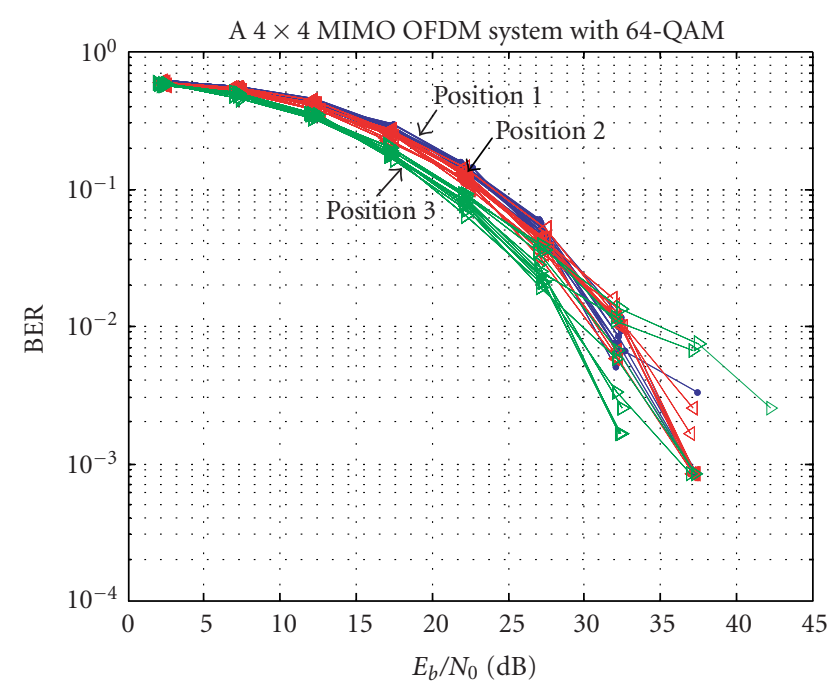

FIgURE 9: The BER-SNR curves measured at the three positions.

It is meaningful to compare the experimental data with the simulation results derived from the MIMO channel model which are applicable for indoor environments. In the paper, we adopt the channel model suggested by the IEEE 802.11 task group [9]. To simulate the testbed configuration at location 3, the following setups are used. Two ULA arrays with four elements spaced by 3 wavelengths are adopted at the transmitter and receiver. The distance between the transmitter and receiver are about 3 meters with non-LOS wireless links. D model is selected representing a typical office or laboratory environment. The pedestrians are moving around at a speed of $1.2 \mathrm{Km} / \mathrm{hr}$, while the fluorescent effects are considered as well. Figure 10 shows the comparisons of the measured BER-SNR curves with simulation results at location 3. The performance match between the experimental data and simulation results validate the efficiency of the MIMO channel model defined by [9] for an indoor environments with the given bandwidth and frequency.

We investigate the degradation of the BER-SNR performance caused by the estimation inaccuracy of MIMO channel due to the finite resolution of ADC/DAC and the unavoidable processing errors. Figure 11 shows the impairments of BER-SNR performance caused by the inaccuracy of channel estimation. Assume that the estimated channel complex gain is $\hat{h}=h+\alpha \zeta$, where the $h$ is the real channel complex gain, $\alpha$ is a factor, and $\zeta$ is a complex Gaussian variable with zero mean and a variance of 1 . The relative channel inaccuracy is defined by $d=\alpha^{2} /|h|^{2} \times 100 \%$. The results show that the MIMO OFDM systems are susceptible to channel estimation inaccuracy. A channel estimate with an inaccuracy of less than $0.01 \%$ is required for a $4 \times 4$ MIMO system.

\section{THE IMPACTS OF CARRIER FREQUENCY OFFSET}

Carrier frequency offset is a common misalignment for wireless communications systems that caused by the frequency

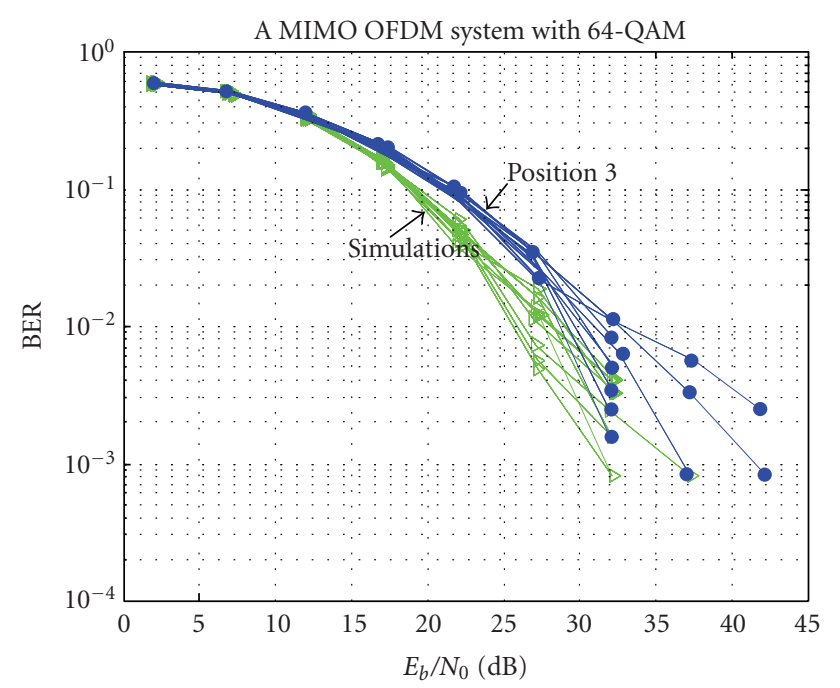

FIgURE 10: The comparison of BER-SNR curves from measurements and simulations.

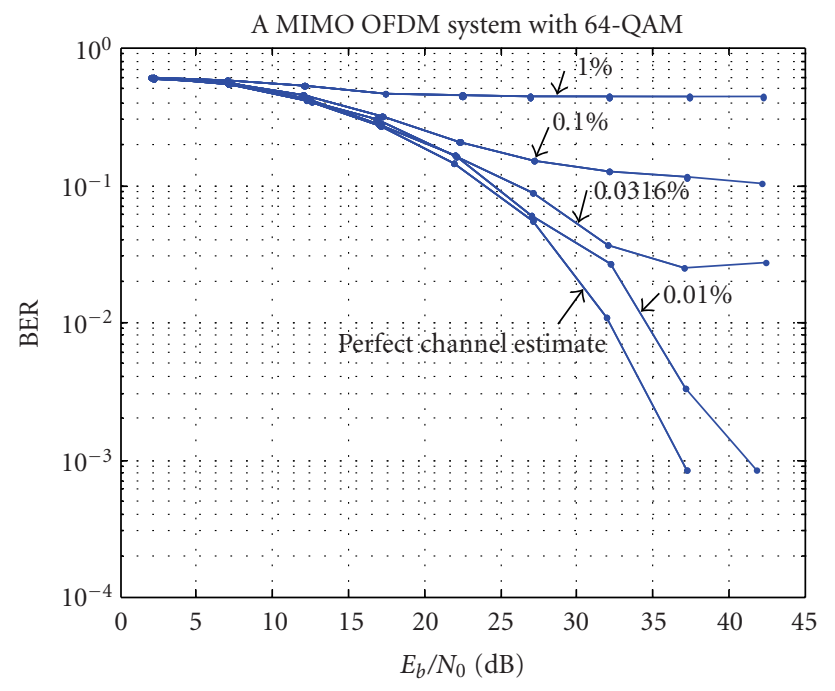

FIGURE 11: The requirements of the accuracy of the channel estimate. (Based on the MIMO channels at position 1.)

drifts between the local oscillators at transmitters and receivers. The frequency offset breaks the orthogonal condition between the subcarriers which decreases the amplitude of the wanted signal and introduces additional intercarrier interferences (ICI). The frequency offset results in an additional signal-to-interference ratio (SIR) equivalently. In the view of the demodulated QAM symbol constellations, the frequency offset leads to rotation of the constellations from their ideal positions in a direction decided by the sign of the frequency offset. Figure 12 gives an example of the demodulated QAM symbols distorted by a carrier frequency offset of 0.05 , where the frequency offset is normalized by the subcarrier spacing. The impairments of carrier frequency offset are distinct from 


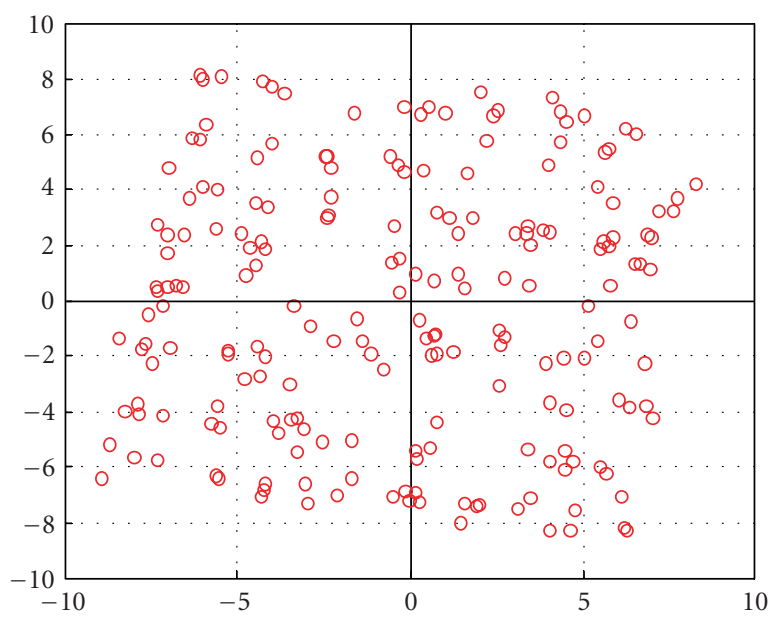

FIGURE 12: The impacts on constellations for 64-QAM systems when a carrier frequency offset of 0.05 is presented.

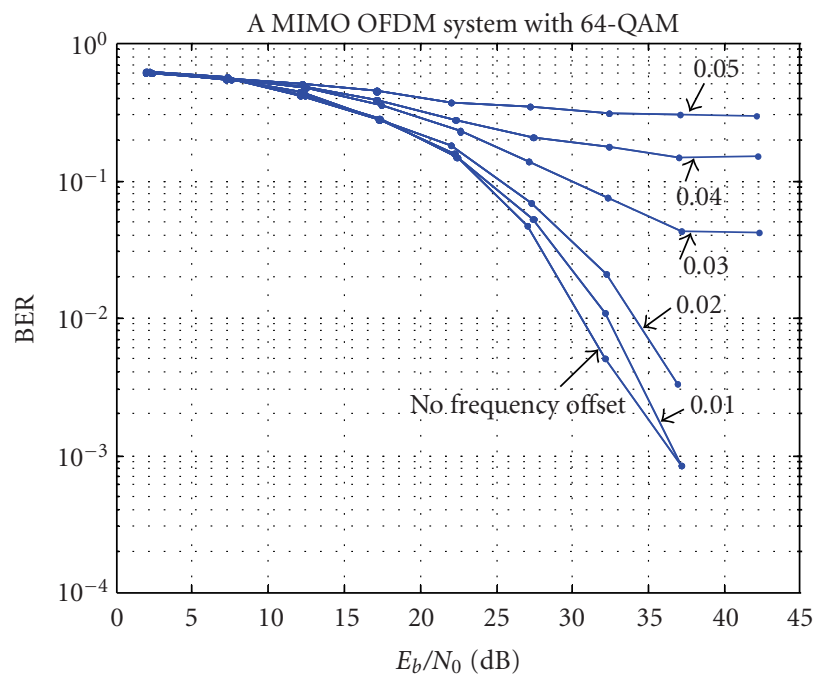

FIGURE 13: The impacts on the BER-SNR curves of the carrier frequency offsets at the position 1 .

Gaussian noise and cannot be compensated for by simply increasing the transmitted power. The receiver has to detect the carrier frequency offset and compensate for the distortions in either frequency domain or time domain. Figure 13 shows the BER-SNR performance degradations against carrier frequency offset. It is easy to see that the BER-SNR curves of MIMO systems demonstrate a high sensitivity to frequency offset when compared to SISO systems. As we can see from Figure 13, the $4 \times 4$ MIMO OFDM systems require frequency synchronization with an offset less than 0.01 , that is $1.367 \mathrm{KHz}$.

\section{ASYMMETRIC MIMO SYSTEM: THE PERFORMANCE OF A $3 \times 4$ MIMO OFDM SYSTEM}

The sensitivity of the performance of MIMO OFDM systems to channel estimation inaccuracy and frequency offset creates

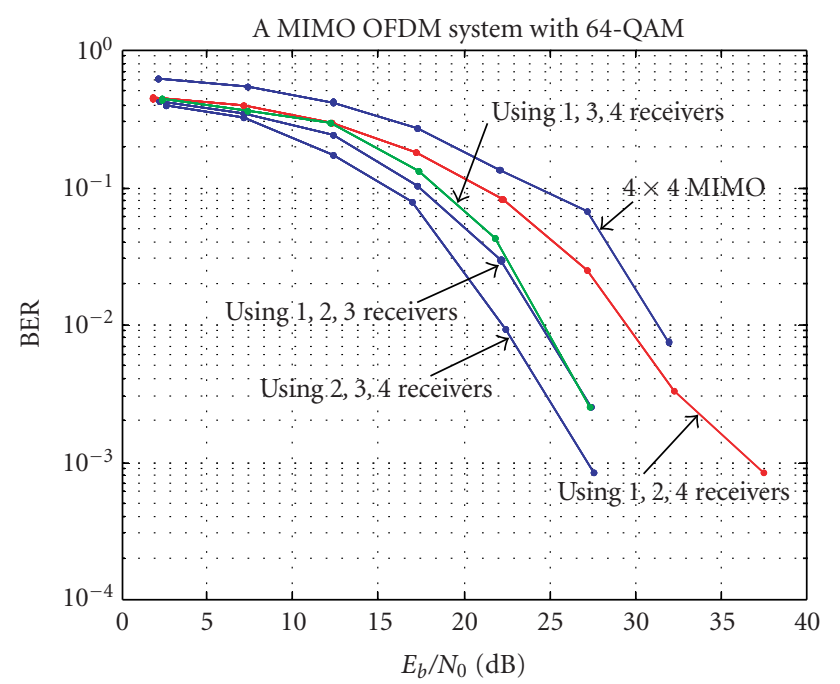

FIGURE 14: The BER-SNR curves of the suggested $3 \times 4$ asymmetric MIMO OFDM system.

challenges to establish low-cost commercial MIMO OFDM systems. In the meantime, the capacities of MIMO systems vary with the MIMO channel statuses. All of these provide the researchers a host of new research topics.

Here we propose an asymmetric MIMO system configuration to take the advantage of redundant receive signals. A three-transmitter four-receiver $(3 \times 4)$ MIMO system is constructed by simply turning off one of the transmitters. In such a case, there are four receive signals, generating four choices to select three of them. The receiver compares and selects the three signals that give a lowest BER. A $3 \times 4$ MIMO OFDM system offers an improved BER-SNR performance statistically compared to a $3 \times 3$ MIMO system with a data rate of $393.75 \mathrm{Mb} / \mathrm{s}$. Figure 14 gives the four BER-SNR curves at location 1 and the one with lowest BER is selected. This is a simple selective diversity scheme for asymmetric MIMO systems.

Furthermore, we introduce a so-called forward estimate BLAST demodulation method to asymmetric MIMO systems. The MIMO channel condition number reflects the system capacity roughly and the computation loads for calculating singular values are much less than the BLAST demodulation. Approximately, the singular values can be acquired during the singular value decomposition (SVD) of the channel matrix, which is part of the processing of channel matrix inversion. For typical order decision feedback (ODF) BLAST method, the computation includes three times of order decision, channel matrix inversion, and matrix multiplication. To avoid three times repeat of the BLAST demodulation, we compare the condition numbers of all the four $3 \times 3$ MIMO combinations before the BLAST modulation and select the configuration with smallest condition number, which is shown in Figure 15. Table 2 lists the four combinations and related condition numbers, where the combination of $\# 2$, \#3, and \#4 receivers give the smallest condition number of 4.5 


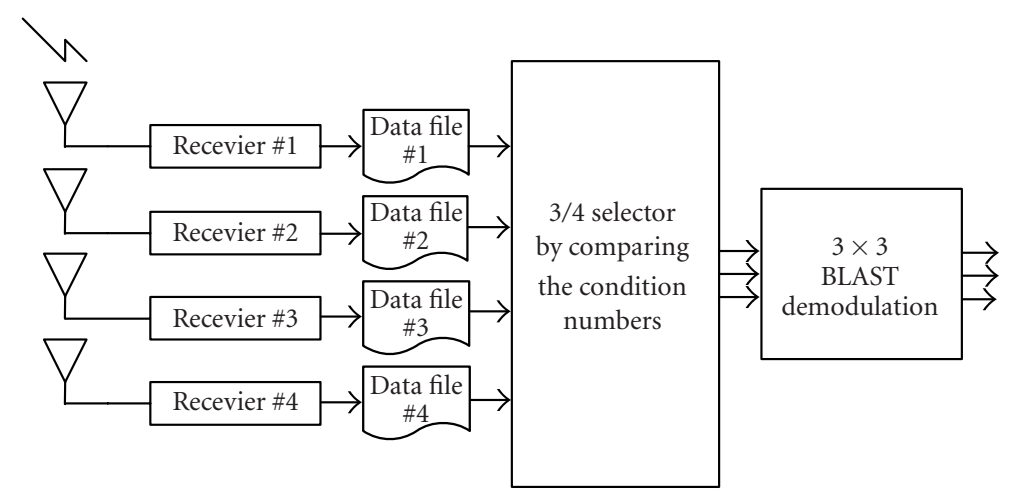

Figure 15: The diagram of the suggested $3 \times 4$ asymmetric MIMO OFDM system.

TABLE 2: The combinations and related condition numbers.

\begin{tabular}{cc}
\hline Selection & Condition number \\
\hline$\# 1, \# 2$, and \#3 receivers & 6.2 \\
$\# 1, \# 2$, and \#4 receivers & 9.3 \\
$\# 1, \# 3$, and \#4 receivers & 7.1 \\
$\# 2, \# 3$, and \#4 receivers & 4.5 \\
\hline
\end{tabular}

and is selected. From the results, the selected configuration provides a gain of 3-10 $\mathrm{dB}$ comparing to the others.

Compared to the other advanced wireless transmission techniques, like coding and adaptive modulation, the asymmetric MIMO scheme is regarded as a cost-effective solution since it only requires one or more additional RF receivers and a selection algorithm. Whereas a powerful coding likely reduces the achievable data rate as well as results in large processing latency. And an adaptive transmission requires complex parameter estimation, prompt, and accurate feedback and duplex channel.

\section{CONCLUSION}

A $4 \times 4$ MIMO OFDM indoor wireless communication testbed is set up, which offers a peak data rate of $525 \mathrm{Mb} / \mathrm{s}$ with a spectral efficiency of 19.2 bits/Hz/s. The experiment results verify the feasibility to achieve extreme high data rate by adopting MIMO and OFDM parallel transmission technologies. The match of the experiment data and simulation validates the efficiency of the channel model defined by IEEE 802.11 study group for indoor MIMO channels at center frequency of $2.435 \mathrm{GHz}$ with a bandwidth about $27 \mathrm{MHz}$.

In the meantime, the experimental results demonstrate the BER-SNR performance of the MIMO OFDM systems is quite fair and susceptible to the various misalignments, such as frequency offset and channel estimation inaccuracy. The enabling technologies, such as coding, diversity, and adaptive transmission, are needed to decrease the BER of MIMO wireless link.

An asymmetric MIMO scheme is proposed as a costeffective way to improve the BER-SNR performance. This scheme is suitable for low-cost commercial products. An efficient scheme that optimally combines all the four received signals will be studied.

\section{REFERENCES}

[1] G. D. Golden, C. J. Foschini, R. A. Valenzuela, and P. W. Wolniansky, "Detection algorithm and initial laboratory results using V-BLAST space-time communication architecture," Electronics Letters, vol. 35, no. 1, pp. 14-16, 1999.

[2] W. Xiang, D. Waters, T. G. Pratt, J. Barry, and B. Walkenhorst, "Implementation and experimental results of a threetransmitter three-receiver OFDM/BLAST testbed," IEEE Communications Magazine, vol. 42, no. 12, pp. 88-95, 2004.

[3] W. Xiang, T. Pratt, and X. Wang, "A software radio testbed for two-transmitter two-receiver space-time coding OFDM wireless LAN," IEEE Communications Magazine, vol. 42, no. 6, pp. S20-S28, 2004.

[4] H. Sampath, S. Talwar, J. Tellado, V. Erceg, and A. Paulraj, "A fourth-generation MIMO-OFDM broadband wireless system: design, performance, and field trial results," IEEE Communications Magazine, vol. 40, no. 9, pp. 143-149, 2002.

[5] R. J. Piechocki, P. N. Fletcher, A. R. Nix, C. N. Canagarajah, and J. P. McGeehan, "Performance evaluation of BLAST-OFDM enhanced Hiperlan/2 using simulated and measured channel data," Electronics Letters, vol. 37, no. 18, pp. 1137-1139, 2001.

[6] M. D. Batariere, J. F. Kepler, T. P. Krauss, S. Mukthavaram, J. W. Porter, and F. W. Vook, "An experimental OFDM system for broadband mobile communications," in Proceedings of 54th IEEE Vehicular Technology Conference (VTC '01), vol. 4, pp. 1947-1951, Atlantic City, NJ, USA, October 2001.

[7] J. W. Wallace, B. D. Jeffs, and M. A. Jensen, "A real-time multiple antenna element testbed for MIMO algorithm development and assessment," in Proceedings of IEEE Antennas and Propagation Society International Symposium, vol. 2, pp. 1716-1719, Monterey, Calif, USA, June 2004.

[8] IEEE P802.16a/D4-2002, "Part 16: Air interface for fixed broadband wireless access systems," 2002.

[9] L. Schumacher, "WLAN MIMO Channel Matlab Program," download information: http://www.info.fundp.ac.be/ lsc/Research/IEEE_80211_HTSG_CMSC/distribution_terms.html. 
Weidong Xiang received his M.S.E.E. and Ph.D. degrees from Tsinghua University, Beijing, China, in 1996 and 1999, respectively. From 1999 to 2004, he worked as a Postdoctoral Fellow and then Research Scientist in the Software Radio Laboratory (SRL) at Georgia Institute of Technology, Atlanta, USA. In September 2004, he joined the ECE Department, University of Michigan, Dearborn, as an Assistant Professor.

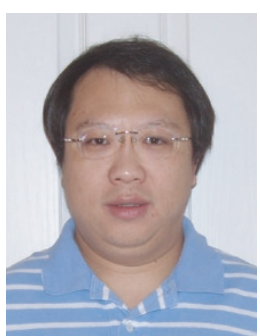
His research interests include high-speed wireless LAN prototype integrating MIMO, OFDM, software radio, and smart antenna, wireless access for vehicular environments (WAVE), ultrawide band (UWB), and real-time wireless control network.

Paul Richardson is an Associate Professor in the Department of Electrical and Computer Engineering, University of Michigan, Dearborn. He is a Principal Investigator for ultrawideband applications with the U.S. Army Research Development and Engineering Center, Warren, Mich, and a Consultant for the United States Marine Corps regarding command and control networks. He received the B.S.E. degree in computer engineering, the M.S.E. degree in computer and electrical engineering, and the Ph.D. degree in systems engineering, all from Oakland University, Rochester, Mich. His interests include embedded real-time systems, vehicular networks and communications systems, and ultrawideband applications.

Brett Walkenhorst received the B.S. and M.S. degrees in electrical engineering from Brigham Young University (BYU), Provo, UT, in 2001. From 2001 to 2003, he worked as a Design Engineer at Lucent Technologies, Bell Laboratories, Denver, Colo. He is currently a Research Engineer at the Georgia Tech Research Institute in Atlanta, Ga. His research interests include signal processing for wireless communications, electromagnetic theory, channel estimation, and neural networks.

Xudong Wang received the Ph.D. degree from Georgia Institute of Technology in 2003. He also received his B.E. and Ph.D. degrees from Shanghai Jiao Tong University, Shanghai, China, in 1992 and 1997, respectively. From 1998 to 2003, he was with the Broadband and Wireless Networking (BWN) Lab at Georgia Institute of Technology. Currently, he is a Senior Research Engineer with Kiyon, Inc., where he leads re-
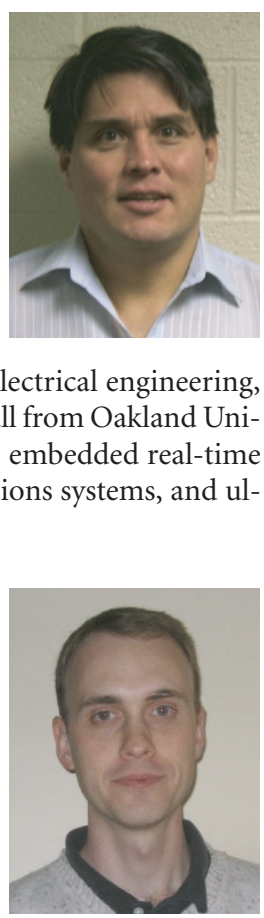
search and development of MAC and routing protocols for wireless mesh networks. His research interests also include software radios, cross-layer design, and communication protocols for cellular, mobile ad hoc, sensor, and ultrawideband networks. He has served as a technical committee member for many international conferences, and has been a technical reviewer for numerous international journals and conferences. He has two patents pending in wireless mesh networks. He is a Member of IEEE, ACM, and ACM SIGMOBILE.

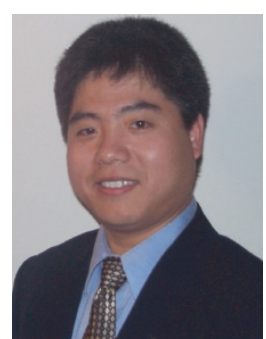

Thomas Pratt received the B.S. degree from the University of Notre Dame, Notre Dame, Ind, in 1985, and the M.S. and Ph.D. degrees in electrical engineering from the Georgia Institute of Technology, Atlanta, in 1989 and 1999, respectively. He heads the Software Radio Laboratory at Georgia Tech, where research has focused principally on MIMO-OFDM, space-time adaptive processing, WLAN interference sup-

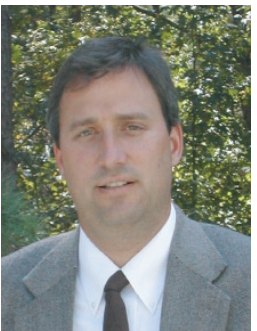
pression, multiple-antenna architectures, channel modeling, and mobile communications. He is a Principal Research Engineer at the Georgia Tech Research Institute. 